\title{
SLICHTER MODES AND LOVE NUMBERS
}

\author{
David. J. Crossley \\ Department of Geological Sciences, McGill University \\ Michael G. Rochester and Z. R. Peng \\ Department of Earth Sciences, Memorial University of Newfoundland
}

Abstract. The recent claim by Smylie [1992] to have detected the Slichter modes of the Earth's inner core rests on what appears to be a remarkable agreement between theory and observation. However the theoretical eigenperiods which are used by Smylie conflict significantly with all previously published periods for the Slichter modes, both for non-rotating and rotating Earth models. A closer examination of the theory used in these calculations reveals the use of static Love numbers to represent the response of the inner core and mantle to dynamics in the liquid core. We here show that the use of dynamic Love numbers restores the eigenperiods to those obtained using standard seismological theory and consequently destroys agreement between these periods and the claimed observations.

\section{Introduction}

The Slichter triplet is a curiosity in the family of Earth's normal modes. Unlike the rest of the seismic normal modes, the restoring force on the inner core is primarily gravitational rather than elastic, and the triplet has never been observed following an earthquake [Rydelek and Knopoff, 1984]. From a classification point of view, it is now generally known as ${ }_{1} S_{1}^{m}$ [cf. Crossley, 1975b], denoting the first overtone $n=1$ of the degree $l=1$ motions.

Adopting the conventions $u \propto e^{i(\omega t+m \phi)}$ for the displacement field $u, \phi$ positive eastward (with rotation) and using positive frequencies $\omega$, it is clear that $m=+1$ is a westward wave motion on a sphere, $m=0$ is axisymmetric and $m=-1$ is eastward. On a rotating sphere, $m=+1$ is therefore retrograde (against rotation) and has a higher frequency (shorter period). The opposite is true for the prograde mode $m=-1$. Curiously, Smylie [1992] adopts the reverse terminology for prograde and retrograde motions. To first order in rotation, the frequency of the $m=0$ wave is unchanged, but second order and higher order perturbation theory also shifts the period of this mode from the non-rotating value.

Because of the high frequency of the elastic normal modes compared to the sidereal rotation frequency, first or second order perturbation theory is usually considered adequate to accurately find the Coriolis splitting of the degenerate eigenfrequencies. Due to the relatively weak gravitational restoring force on the inner core, perturbation theory is less applicable for the long period (several hours) of the

Copyright 1992 by the American Geophysical Union.

Paper number 92GL01574

0094-8534/92/92GL-01574\$03.00
Slichter modes [Crossley, 1975b; Smith 1976; Dahlen and Sailor, 1979].

Love numbers have generally been used to represent the elastic response of the Earth to tidal forcing, and as such are generally considered to be static, representing the response of the Earth at periods much longer than the elastic normal modes. However, as pointed out by Pekeris and Accad [1972], the tidal Love numbers do in fact vary with frequency at long periods (several hours) for an Earth model with a stably stratified liquid core, due to resonances at the periods of internal gravity waves in the fluid. Considerable simplification can be made in the dynamics of the liquid core by using load Love numbers to represent the response of the inner core and mantle [Smylie, 1988; Rochester and Peng, 1990; Crossley et al., 1991].

The Love numbers computed for $l=1$, particularly those for the inner core boundary (ICB), have resonances close to those of the Slichter periods for the whole Earth and therefore static values cannot be used to represent the response of the inner core and mantle. Indeed on physical grounds it should be immediately obvious that, because the Slichter modes are primarily motions of the inner core, one cannot focus on the dynamic response of the liquid outer core and ignore the the inertia term in the computation of the ICB Love numbers when these Love numbers explicitly incorporate the inner core oscillation. Unfortunately, Smylie [1988] missed this important point when he assumed that the inertial terms in the inner core and mantle were insignificant, and he took the deformation field therein to be static.

Rochester and Peng [1990] first discussed the calculation of the Slichter modes using the variational procedure with the subseismic approximation (SSA) and dynamic Love numbers for the inner core and mantle. Here we recompute the Slichter periods for a variety of Earth models using both direct integration and Love numbers. In order to reproduce Smylie's periods as closely as possible, we also used the subseismic approximation in two related forms. SSA-1 refers to the application of the subseismic approximation only in the body of the liquid core [Smylie and Rochester, 1981] and using the boundary conditions that apply for the full system. SSA-2 refers to a further stage of approximation, that used by Smylie et al. [1990], in which the SSA is applied also to the boundary conditions [cf. Crossley and Rochester, 1992].

\section{$\triangle \rho_{I C B}$ and $N^{2}$}

We begin by noting the relation between the density jump at the ICB $\left(\Delta \rho_{I C B}\right)$ and the non-rotating Slichter eigenperiod $T_{0}$. We construct, beginning with PREM [Drie- 
wonski and Anderson, 1981], a suite of Earth models with liquid cores of different buoyancy periods $T_{B}=2 \pi / N$, where $N$ is the Brünt Väisälä frequency, and different values of $\Delta \rho_{I C B}$. In each model, the seismic velocities are unchanged throughout the Earth and for each new $T_{B}$ the mass of the Earth is also maintained. Then we integrated the equations of motion directly and obtained values of $T_{0}$. The starting conditions at the Earth's centre have to be correctly satisfied to allow displacements of the inner core about the fixed centre of mass [Crossley, 1975a].

The results (Figure 1) indicate that outer core stability plays a significant role only when $\Delta \rho_{I C B}<0.4 \mathrm{~g} \mathrm{~cm}^{-3}$ and that a strongly stable stratification can provide a gravitational restoring force even for negative density contrasts. For a neutral core, the Slichter period becomes infinite as $\Delta \rho_{I C B} \rightarrow 0$. We show our computed periods for 1066A [Gilbert and Dziewonski, 1975], PREM and CORE11 [Widmer et al., 1988]. Also plotted are the periods for DG579, an unlisted model used by Smith [1976], and model 71BG of Smylie [1992], using respectively their quoted values of $\Delta \rho_{I C B}$ and $T_{0}$. Smylie's eigenperiods for $71 \mathrm{BG}, 1066 \mathrm{~A}$ and CORE11 are much below the curve along which all other results are grouped.

An Earth model with a period $T_{0}$ as short as that quoted for $71 \mathrm{BG}$ requires $\Delta \rho_{I C B}=1.26 \mathrm{~g} \mathrm{~cm}^{-3}$. This value is considered seismologically extremely unlikely from an analysis of a suite of elastic normal modes studied by Masters and Shearer [1990, Figure 1], which favours $0.475 \mathrm{~g} \mathrm{~cm}^{-3}$.

\section{Dynamic Love numbers}

As discussed by Smylie et al. [1990], Crossley et al. [1991] and Rochester and Peng [1992], the use of Love numbers considerably simplifies core dynamics in the cases where the boundaries are elastic and/or the gravity effect at the surface (through the mantle eigenfunctions) is required.

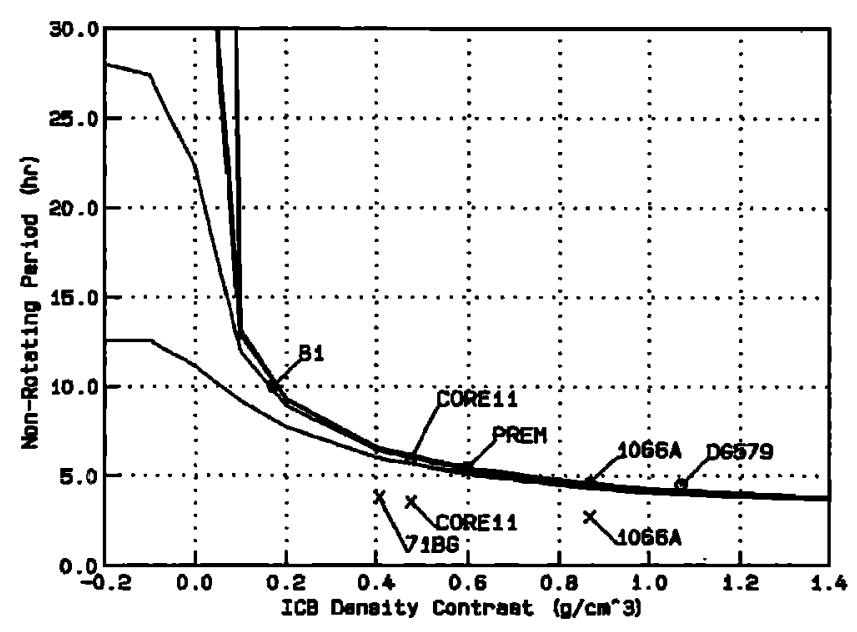

Fig. 1. Non-rotating Slichter eigenperiods for several Earth models. The 4 solid curves, from bottom to top, were computed using modified liquid cores of buoyancy periods $6 \mathrm{hr}$, $12 \mathrm{hr}, 18 \mathrm{hr}$ and neutral respectively. The circles and crosses are periods computed for seismic Earth models by us and by Smylie [1992] respectively.
Load Love numbers can be regarded as matching constants connecting the dynamic variables at the ICB and core mantle boundary (CMB). The 6th order equations are integrated for three trial solutions throughout the inner core, from the Earth's centre to the ICB. On applying the appropriate boundary conditions at the ICB, one obtains two independent pairs of load Love numbers, from which any of the regular variables (radial displacements, stresses or gravity variables) can be expressed. These Love numbers are then stored for future use.

The process is repeated for the mantle, integrating from the CMB to the Earth's surface where traditional boundary conditions are applied. Again two more pairs of independent Love numbers are obtained and stored. At this point, one goes back and integrates throughout the fluid core, with two trial solutions at a trial eigenfrequency for the four independent equations in the fluid, using the Love numbers where appropriate to construct the discriminant (based on continuity of radial displacement and normal stress at the CMB) for each trial eigenfrequency. The key question is whether or not the load Love numbers need be re-computed each time the outer core eigenfrequency is re-adjusted (dynamic Love numbers), or whether static (zero frequency) Love numbers computed once only are sufficient.

In principle, all Love numbers are dynamic since the equations integrated in the solid normally contain the inertial term $\omega^{2} u$. Load Love numbers exhibit resonances that reflect eigensolutions of the restricted problem being addressed. For example, the ICB Love numbers have a resonance wherever there is a true eigensolution of a realistic inner core bathed in an infinite fluid of uniform density equal to the density on the fluid side of the ICB, since this density was embedded in the boundary condition used at the ICB to generate the Love numbers. Similarly, a CMB Love number shows resonances at the eigensolutions of a realistic shell (the mantle) filled inside with a fluid of uniform density equal to that on the fluid side of the CMB.

It can be seen in Figure 2 that in the neighbourhood of an isolated resonance $\omega_{L}$ the Love numbers are of the form $h(\omega)=h(0)+h_{L}\left[\left(1-\omega^{2} / \omega_{L}^{2}\right)^{-1}-1\right]$ where $h(\omega)$ is the dynamic Love number and $h(0)$ is the static Love

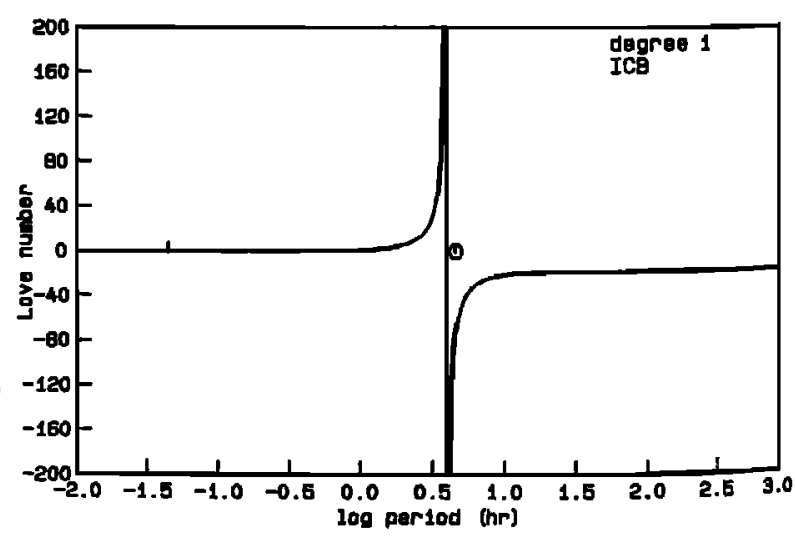

Fig. 2. The $l=1$ ICB Love number for $\chi$ in the period range $10 \mathrm{sec}$ to $1000 \mathrm{hr}$. The whole-Earth Slichter eigerperiod of $4.599 \mathrm{hr}$ (circle) lies almost on top of the large inner core resonance at $3.995 \mathrm{hr}$. 
number. Only if $\omega^{2} \ll \omega_{L}^{2}$ may we take $h(\omega) \simeq h(0)$. The above expression for the Love number does not have to be programmed into the computations, but arises naturally as a result of including the inertial term in the equations of motion.

We can now appreciate the significance of using dynamic Love numbers whenever the anticipated eigenfrequency of the complete system is close to a Love number resonance. Since the ICB Love number resonance for $1066 \mathrm{~A}$ is at $T_{L}=$ $3.995 \mathrm{hr}$, it is close to the anticipated period of $T=4.599$ $\mathrm{hr}$ (Figure 2). Consequently it is essential to use dynamic Love numbers for the Slichter modes.

Table 1 shows dynamic and static Love numbers for $\chi$, the reduced potential and $d \phi / d r$, the derivative of the gravity potential, for 3 Earth models and compares the results to those of Smylie [1992]. We note first that the degree 1 Love numbers are extremely sensitive to the details of the numerical methods used in the integration, which is suffcient to explain the slight discrepancies between the various authors for the same calculation. With this in mind, it is quite clear that important conclusions can be drawn. First, we have recovered Smylie's static Love numbers for $1066 \mathrm{~A}$ and CORE11. This indicates that all authors agree, within numerical error, with the method of integrating the equations of motion in the limit of zero frequency.

Second, independent calculations (Crossley, Peng) confirm the Love numbers computed at the periods of the respective non-rotating Slichter modes and are significantly different from the static values, as to be expected from Figure 2. This difference explains the bulk of the discrepancy between Smylie's computed periods and our new calculations which agree with standard seismological theory.

\section{Coriolis Splitting}

Before we can compare our final results to those of Smylie [1992], we have to tackle the question of Coriolis splitting. Dahlen and Sailor [1979] elegantly summarized the results of second order perturbation theory in their analysis of elastic overtones including, fortunately, the Slichter mode. Additionally, Crossley [1975b], Smith [1976], Melchior et al. 1988] and Rochester and Peng [1992] all gave results

TABLE 1 - Love numbers of degree 1

\begin{tabular}{lrrrl}
\hline model & $\begin{array}{r}\text { period } \\
(\mathrm{hrs})\end{array}$ & $\begin{array}{r}\chi \\
(I C B)\end{array}$ & $\begin{array}{r}d \phi / d r \\
(I C B)\end{array}$ & $\begin{array}{l}\text { Type, } \\
\text { Author }^{(1)}\end{array}$ \\
\hline $1066 \mathrm{~A}$ & 4.59919 & -72.9780 & 5.2429 & $\mathrm{D}, \mathrm{C}$ \\
& 4.60382 & -72.8092 & 5.2294 & $\mathrm{D}, \mathrm{P}$ \\
& 1000.0 & -18.0459 & 1.2882 & $\mathrm{~S}, \mathrm{C}$ \\
CORE11 & 5.94692 & -17.8629 & -1.2755 & $\mathrm{~S}, \mathrm{~S}^{(2)}$ \\
& 1000.0 & -28.8533 & 4.1220 & $\mathrm{D}, \mathrm{C}$ \\
& $\infty$ & -28.0179 & -1.1652 & $\mathrm{~S}, \mathrm{C}$ \\
PREM & 5.42063 & -83.3317 & 4.2370 & $\mathrm{~S}, \mathrm{~S}^{(2)}$ \\
& 5.41190 & -82.5272 & 4.2226 & $\mathrm{D}, \mathrm{P}$ \\
& 1000.0 & -23.8203 & 1.2037 & $\mathrm{~S}, \mathrm{C}$ \\
\hline
\end{tabular}

(1) Type $\mathrm{D}=$ dynamic, $\mathrm{S}=$ static

Author C = Crossley, $\mathrm{P}=$ Peng, $\mathrm{S}=$ Smylie et al. [1990],

but note the different values quoted in Smylie et al. [1992]

(2) The period used by Smylie is not known; his Love numbers for $d \phi / d r$ are defined with the opposite sign to us. from a more complete theory that includes higher order terms in the expansion of the displacement field, necessary for long period oscillations on a rotating Earth.

Perturbation theory expands the displacement field in terms of $(\Omega / \omega)^{k}$, where $\Omega$ is the sidereal rotation frequency. First order theory $(k=1)$ includes the Coriolis force in the basic spheroidal term of the spherical harmonic expansion, dubbed 'self-coupling' by Crossley [1975b]. Second order $(k=2)$ includes the next toroidal term $T_{l+1}^{m}$ which is the basis of most of the rotating eigenperiods quoted in the references above. Recently, using SSA-1, Rochester and Peng [1992] have extended the coupling chain to degree 5, i.e. the displacement field is represented by harmonics $S_{1}^{m}$ $\ldots S_{5}^{m}$. Smylie [1992] uses SSA-2 with an unknown number of terms to expand the variable $\chi$ at the ICB and CMB.

Our final results for the Slichter eigenperiods are given in Table 2. We denote by $T_{P}$ the prograde $(m=-1)$ mode, $T_{R}$ the retrograde $(m=+1)$ mode, and $T_{C}$ the central $(m=0)$ peak. Our split eigenperiods are computed using the second order perturbation formulae of Dahlen and Sailor [1979].

Notice that for the full equations, direct and Love number methods yield identical results, showing correct implementation of the algorithms. Necessarily, the dynamic Love number calculations also agree with previous values, allowing for the sensitivity of the Slichter periods to details of the integration method. SSA-1 yields little change from the full equations, whereas SSA-2 underestimates the correct eigenperiod by about $10 \%$ due to problems with the SSA at the CMB [Crossley and Rochester, 1992]. The periods using static Love numbers are $40 \%$ too low.

Results for several Earth models are plotted in Figure 3, which is analogous to that shown by Smylie [1992]. Note that with dynamic Love numbers, not only would the 'observed' eigenperiods require an unacceptably high ICB density contrast, but also the splitting is greater than that obtained theoretically and observationally by Smylie [1992, equation (1)]. This implies that if the the observational triplet identified by him were to be confirmed, the peaks are unlikely to be associated with a co-rotating physical oscillation of the Earth.

TABLE 2 - Slichter mode eigenperiods, model 1066A

\begin{tabular}{ccccl}
\hline$T_{0}$ & $T_{\tau}{ }^{(1)}$ & $T_{c}^{(1)}$ & $T_{p}^{(1)}$ & $\begin{array}{l}\text { Equations, } \\
\text { Method, Author }\end{array}$ \\
\hline 4.59920 & 4.12843 & 4.53384 & 5.01400 & F,D,C \\
4.59920 & 4.12843 & 4.53384 & 5.01400 & F,LN,C \\
4.5984 & & & & F,D,MD \\
4.59759 & 4.12759 & 4.53222 & 5.01135 & SSA-1,D,C \\
4.59760 & 4.12760 & 4.53222 & 5.01146 & SSA-1,LN,C \\
4.49940 & 4.04970 & 4.43806 & 4.89647 & SSA-2,LN,C \\
2.62518 & 2.46200 & 2.61407 & 2.78137 & F,SLN,C \\
2.40404 & 2.23077 & 2.39882 & 2.58229 & SSA-1,SLN,C \\
2.75079 & 2.58456 & 2.73665 & 2.90511 & SSA-2,SLN,C \\
2.7141 & 2.6035 & 2.7023 & 2.8247 & SSA-2,SLN,S \\
\hline
\end{tabular}

(1) Periods in hours. Rotating eigenperiods are by 2nd order splitting except for Smylie (full rotation).

(2) Equations F = full, SSA-1, SSA-2; Method D = direct, LN = dynamic Love number, SLN = static Love number; Author $\mathrm{C}=$ Crossley, $\mathrm{MD}=$ Masters, Dahlen (independent personal communications, May 1992), S = Smylie. 


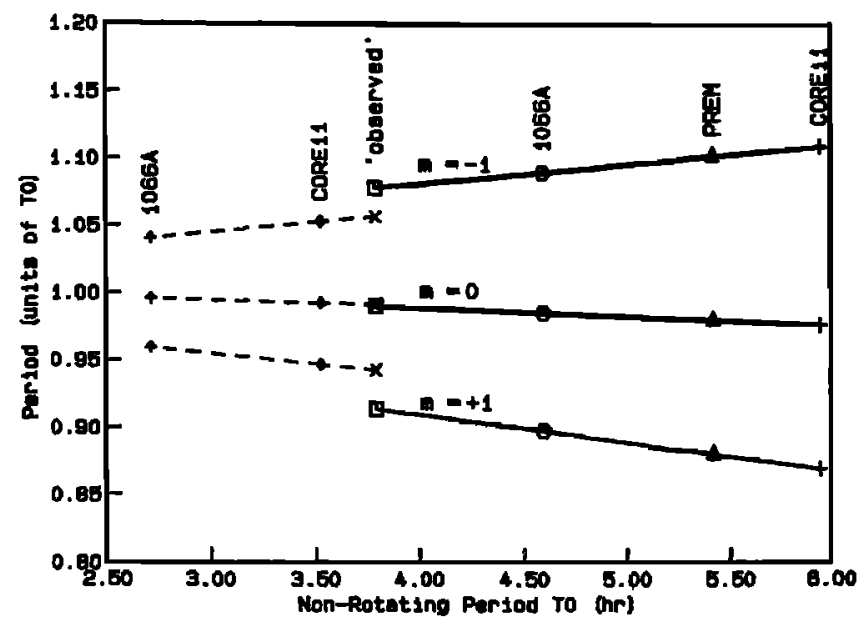

Fig. 3. Coriolis splitting of Slichter modes for the prograde $(m=-1)$, central $(m=0)$ and retrograde $(m=+1)$ components at the top, centre and bottom respectively. The solid lines join the periods computed using second order perturbation theory (at this scale almost identical to coupling to degree 5 in the fluid) and show models $1066 \mathrm{~A}$ (circles), PREM (triangles) and CORE11 (crosses). The dashed lines show the splitting according to Smylie [1992] for 1066A (arrows), CORE11 (diamonds) and the claimed observations (crosses). The squares show a model in which we modified CORE11 to have an ICB density jump of 1.26 $\mathrm{g} \mathrm{cm}^{-3}$, calculated to provide agreement with the period of the $m=0$ mode for the claimed observations; evidently the true splitting ( $m= \pm 1$ periods) disagrees with that computed by Smylie.

\section{Summary}

We have verified Smylie's eigenperiods to within $2 \%$, indicating to our satisfaction that we have correctly understood his numerical procedures. We have therein demonstrated that the use of static rather than dynamic Love numbers is the main reason he obtains significantly different Slichter eigenperiods from published and here reconfirmed values. The substantial correction necessary to Smylie's eigenperiods leads to complete disagreement with the periods he identifies in the data and destroys his claim to have detected the Slichter modes.

Acknowledgements. We wish to thank Jacques Hinderer, Olivia Jensen and Wen-jing Wu for discussions and Tony Dahlen and Guy Masters for re-computing the Slichter periods as an independent check. This research was supported by The Natural Sciences and Engineering Research Council of Canada.

\section{References}

Crossley, D. J., The free-oscillation equations at the centre of the Earth, Geophys. J. Roy. astr. Soc., 41, 152-163, 1975a.

Crossley, D. J., Core undertones with rotation, Geophys. J. Roy. astr. Soc., 42, 477-488, 1975b.

Crossley, D. J., J. Hinderer and H. Legros, On the excitation, detection and damping of core modes, Phys. Earth Planet. Int., 68, 97-116, 1991.

Crossley, D. J., and M. G. Rochester, Simple core undertones, Geophys. J. Roy. astr. Soc., 60, 129-161, 1980.

Crossley, D. J., and M. G. Rochester, The subseismic ap- proximation in core dynamics, Geophys. J. Roy. astr. Soc., 108, 502-506, 1992.

Dahlen, F. A., and R. V. Sailor, Rotation and elliptical splitting of the free oscillations of the Earth, Geophys. J. Roy. astr. Soc., 58, 609-623, 1979.

Dziewonski, A.M., and D. L. Anderson, Preliminary reference Earth model, Phys. Earth Planet. Int., 25, 297-356, 1981.

Gilbert, F., and A. M. Dziewonski, An application of normal mode theory to the retrieval of structural parameters and source mechanisms from seismic spectra, $P$ hil. Trans. Roy. Soc. London, Ser. A, 278, 187-269, 1975.

Masters, G., and P. Shearer, The density and shear velocity contrast at the inner core boundary, Geophys. J. Int, 102, 491-498, 1990.

Melchior, P., D. J. Crossley, V. P. Dehant and B. Ducarme, Have inertial waves been identified from the Earth's core ?, in Structure and Dynamics of Earth's Deep Interior, Geophysical Monograph 46, IUGG Volume 1, edited by D.E. Smylie and R. Hide, Pp. 1-12, AGU, Washington, 1988.

Pekeris, C. L., and Y. Accad, Dynamics of the liquid core of the Earth, Phil. Trans. Roy. Soc. London, Ser. A, 273, 237-260, 1972.

Rydelek, P. A., and L. Knopoff, Spectral analysis of gapped data: search for mode ${ }_{1} S_{1}$ at the South Pole, J. Geophys. Res., 89, 1899-1902, 1984.

Rochester, M. G., and Z. R. Peng, The Slichter mode revisited: a test of the subseismic approximation, EOS, Trans. Am. Geophys. Un., 71, 1479-, 1990.

Rochester, M. G., and Z. R. Peng, The Slichter modes of the rotating Earth: a test of the subseismic approximation, submitted to Geophys. J. Int., 1992.

Smith, M. L., Translational inner core oscillations of a rotating, slightly elliptical Earth, J. Geophys. Res., 81, 3055-3065, 1976.

Smylie, D. E., Variational calculation of core modes of the Earth, in Structure and Dynamics of Earth's Deep In. terior, Geophysical Monograph 46, IUGG Volume 1, edited by D. E. Smylie and R. Hide, pp. 23-28, AGU, Washington, 1988.

Smylie, D. E., The inner core translational triplet and the density near Earth's center, Science, 255, 1678-1682, 1992.

Smylie, D. E., X. Jiang, B. J. Brennan and K. Sato, Numerical calculation of modes of oscillation of the Eartb's core, Geophys. J. Int., 108, 465-490, 1992.

Smylie, D. E., and M. G. Rochester, Compressibility, core dynamics and the subseismic wave equation, Phys. Earth Planet. Int., 24, 308-319, 1981.

Smylie, D. E., A. M. K. Szeto and K. Sato, Elastic boundary conditions in long-period core oscillations, Geophys. J. Int., 100, 183-192, 1990.

Widmer, R., G. Masters and F. Gilbert, The spherical Earth revisited, EOS, Trans. Am. Geophys. Un., 69, $1310,1988$.

David. J. Crossley, Department of Geological Sciences, McGill University, 3450 University St., Montréal, Québéc H3A 2 A7.

Michael G. Rochester and Z. R. Peng, Department of Earth Sciences, Memorial University of Newfoundland, St. John's, Newfoundland A1B 3X7.

(Received: June 2, 1992;

Accepted: June 28, 1992.) 